\title{
Understanding the nature of science through COVID-19 reports
}

\author{
Teaching students the nature of science can be difficult, but the COVID-19 pandemic offers a \\ range of teachable examples in a form of 'living textbook', explains Wei-Zhao Shi.
}

U nderstanding the nature of science is a crucial component of scientific literacy and an important objective of science education. Students with a more-informed understanding of the nature of science are better positioned for higher achievement in science courses, and this understanding also profoundly affects their decision-making in the complex contexts of socioscientific issues that they encounter throughout life. Most students understand science as what is printed in textbooks, and what is included on associated worksheets and in verification-type laboratory exercises. Although there have been suggestions that teachers should help students to learn the nature of science through research articles, there is little guidance on how to do this. Scientific progress as reflected in reports and public debate during the COVID-19 pandemic offers vivid examples of teachable aspects of the nature of science.

Science is subjective. Although bats are recognized as natural hosts of coronaviruses, intermediate hosts have not been identified. Finding possible intermediate hosts of coronaviruses is imperative to prevent the future spread of epidemics. Some researchers suggested that a species of snake was the most plausible intermediate host of SARS-CoV-2, because the codon usage bias of this virus is most similar to the DNA of snakes. Others felt this inference was implausible, and some highlighted that the spread of viruses from poikilotherms to endotherms is considered to be rare. This exemplifies that scientists arrive at their conclusions in different ways, influenced by their prior knowledge, experience, beliefs and expectations.

There is no single scientific method: science involves creativity. One of most common misconceptions that students hold about science is that the same step-by-step scientific method is adopted by all research scientists in all cases. In fact, scientists use a variety of methods to produce fruitful results. The conventional way of detecting potential hosts and pathogenicity of new viruses is on the basis of their sequence similarity to known viruses, either by building a phylogenetic tree or using BLAST.
However, some researchers used the method DeepHoF (deep-learning-based host finder), developed on the basis of a deep-learning algorithm, to predict the host of COVID-19.

The same context has different effects on different branches of science. Because of the COVID-19 pandemic, people in most countries spend more time at home. The reduction of noise caused by human movement is good news for geophysicists. Seismic noise has decreased, allowing detectors to sense smaller earthquakes. Conversely, some large-scale physical experimental equipment has been negatively affected by the fact that no one was maintaining or operating it, which has resulted in the inability to resume work. For example, the Italian Virgo gravitational wave detector was forced to shut down temporarily; the same fate befell the US National Ignition Facility (the world's largest laser) in California.

The response to science is affected by power structures, and political, social and cultural contexts. Early in the pandemic, the government of China made the unprecedented decision to seal off the city of Wuhan from all outside contact to contain the spread of the novel coronavirus outbreak. Italy announced similar measures, blocking travel from and to the entire northern region of Lombardy and 14 provinces (such as Veneto), and putting lockdown measures in place. By contrast, at the same time the UK government allegedly intended to manage - rather than suppress - the spread of the disease through the population, effectively accepting 'herd immunity' through infection. Another example is the adoption of mask-wearing mandates. Even before the pandemic, it was common in East Asian countries for individuals to wear masks to reduce the risk of infection, but in the West requirements to wear masks in public places were initially seen as futile and met with scepticism. Currently, mask wearing is standard in public places in many European countries. There are also cultural differences in the choice of treatment. According to the history of Chinese epidemics, 321 epidemics occurred from the Western Han Dynasty to the end of the Qing
Dynasty (a period of more than 2,000 years); people in China have relied on traditional Chinese medicine for protection for a long time. Traditional Chinese medicine has both medical and cultural attributes. It is mainly influenced by ancient Chinese philosophy, such as yin-yang, 'five agents' (wuxing) and the 'unity of the heaven and humanity'. Traditional Chinese medicine is usually made up of a variety of herbs, whereas Western medicine is made by chemical synthesis. In China, patients with COVID-19 who display mild symptoms are mainly treated with traditional Chinese medicine, whereas those with severe symptoms or who are critically ill are treated with integrated traditional Chinese and Western medicine.

Modern scientific research relies extensively on advanced technology. The earliest version of the novel coronavirus genome sequence was submitted to the NCBI GenBank database by researchers before 10 January 2020. This speed is mainly due to high-throughput sequencing technology. In turn, scientific research also promotes the development of technology. Telemedicine and remote monitoring require real-time collection and analysis of data; 5G technology (with the advantages of large capacity and low delay) has been rapidly promoted and applied.

We will never get the majority of our students to understand science in detail, but we may be able to instil some understanding of how science works. Students typically appreciate the 'here and now' dimension of present cases; the intrinsic topicality of COVID-19-related science therefore turns scientific reports into 'living textbooks'.

\section{Wei-Zhao Shi (D) $ه$ \\ Graduate School of Education, Peking University, Beijing, China. \\ $凶_{e-m a i l: s h i w z @ p k u . e d u . c n}$}

Published online: 7 February 2022 https://doi.org/10.1038/s41562-022-01303-Z

Competing interests

The author declares no competing interests. 